ments of actin + tropomyosin and of actin + tropomyosin $+\mathrm{TN}-\mathrm{T}+\mathrm{TN}-\mathrm{I}$ and formed them into paracrystals by the addition of $\mathrm{Mg}^{2+}$. From the digital scans of micrographs of the paracrystals they selected one filament at a time and reconstructed it in three dimensions. From many such reconstructions the best averages were taken and the two types of filament compared. In the first type of filament the tropomyosin may be clearly distinguished running near the centre of each of the grooves between actin strands. When the TN-T and TN-I are added, however, the tropomyosin is displaced through about $1 \mathrm{~nm}$ and becomes much more closely integrated with each actin strand.

From observations of changes in the X-ray diffraction pattern of living muscle at rest and during contraction, several authors have proposed that tropomyosin moves in the actin grooves when the muscle is stimulated. This leads naturally to the proposal that inhibition is caused by blocking by tropomyosin of the sites of actin that interact with myosin. On stimulation of the muscle, increased $\mathrm{Ca}^{2+}$ concentration is thought to cause a conformational change in troponin with a consequent displacement of tropomyosin towards the centre of each actin groove and removal of the inhibition. Since the distribution of troponin is discrete along the thin filament (one troponin for every seven actin subunits), whereas that of tropomyosin is continuous, the function of the latter is both to mediate and to amplify the effect of the former.

Because only the amplitudes and not the phases of reflections in the X-ray diffraction patterns are recorded, the tropomyosin movement model cannot be substantiated from the whole-muscle studies alone. This is where the electron microscope work proves so useful. The direct determination by Wakabayashi et al. of a tropomyosin shift under the influence of TN-I is therefore an important advance in understanding thin filament control.

The simplicity of the steric blocking mechanism is appealing, but not necessarily convincing, and it may only be a part of a system which involves other subtler allosteric interactions. It has also still to be demonstrated that in the so-called inhibitory position, the tropomyosin does actually cover the actin site where myosin would attach. There is still work to be done then. Moreover, evidence has recently been accumulating for a $\mathrm{Ca}^{2+}$-sensitive regulatory role in the thick as well as in the thin filaments of vertebrate muscles. The full picture is bound to be complex and even the distinction between the process and regulation of contraction may become less clear-cut.

\section{Interferon at Warwick}

from Mike Clemens

Interferon seems to be a popular subject this summer. The most recent of several meetings on it was an EMBO Workshop held at the University of Warwick on July 29-31 in which much new information concerning the induction, purification and mechanism of action of this anti-viral protein was presented.

IT is well known that the synthesis of interferon can be induced in cells not only by intact viruses but also by synthetic double-stranded polynucleotides. But it is not at all clear whether the latter have to enter cells or act at the surface niembrane. D. Hutchinson (University of Warwick) gave a critical account of the evidence in favour of the latter conclusion. In spite of th. almost inevitable solubilisation of some double-stranded RNA which occurs when cells are incubated with this inducer covalently bound to inert supports, direct action at the cell surface seems likely. The immediately subsequent events are still a mystery but considerable progress has been made in identifying the messenger RNA for interferon in cells exposed to doublestranded RNA. P. Pitha (Johns Hopkins University) showed that poly(A)containing RNA from induced human fibroblasts was translated both in mouse cell-free systems and in intact frog oocytes to give products with human interferon activity. No interferon mRNA could be detected in uninduced cells and it seems that regulation of interferon production may well occur at the transcriptional level. D. Burke (University of Warwick) presented evidence that the inhibitor of transcription camptothecin prevents induction of interferon by virus but not by double-stranded RNA, but it is not yet known whether a virus-directed or a host-directed transcriptional event is involved in this effect.

The most controversial area of interferon research concerns its mechanism of action. This has recently been studied by $M$. Revel (Yale University) using somatic cell hybrids containing a limited number of human chromosomes. The requirement for chromosome 21 for sensitivity of cells to human interferon is now thought to be because of a specific membrane receptor for interferon coded by this chromosome, rather than an intracellular 'anti-viral protein'. Studies with human fibroblasts monosomic or trisomic for chromosome 21 have confirmed this conclusion.

The events that occur between inter- action of interferon with its receptor and inhibition of virus replication remain something of a mystery. There was general agreement that protein synthesis is a major target for interferon action but D. Metz (National Institute for Medical Research) produced convincing evidence that, in the case of monkey cells infected with SV40 virus, there is selective inhibition of viral RNA synthesis or enhanced nuclear turnover of viral transcripts after interferon treatment. The recently discovered process of methylation of the $5^{\prime}$ ends of newly synthesised mRNA may also be a target for interferon dction. P. Lengyel (Yale University) reported that the rate and extent of methylation of reovirus mRNAs in interferon-treated cell extracts was only half that seen in control extracts. However, A. Ball (University of Connecticut) stated that, in an interesting coupled transcription-translation system, methylation of newly synthesised vesicular stomatitis virus RNA is not impaired in the presence of extracts of interferon-treated chick embryo cells. In the same incubations translation of this RNA was severely inhibited compared to its translation in control cell extracts.

A possible clue to the mechanism of inhibition of translation came from the observation by I. M. Kerr's laboratory (NIMR) that interferon-treated L-cell extracts are more sensitive to inhibition by double-stranded RNA than control extracts. T. Hunt (University of Cambridge) described some recent experiments demonstrating that, in reticulocyte cell-free systems, double-stranded RNA causes the ATP-dependent phosphorylation of an initiation factor. However several groups have shown that polypeptide chain elongation as well as initiation is impaired by interferon treatment so that the situation is rather complex. Lengyel and Ball both stated that the established ability of tRNA to reverse the interferonmediated inhibition of translation is not observed under certain conditions and the physiological relevance of this effect must therefore remain open to doubt. It would be a brave person who predicts, on the basis of present knowledge, just how interferon prevents viral replication within animal cells.

\section{Correction}

In the article by Donald Boulter "Breeding for Protein Yield and Quality" (Nature, 256, 168; 1975) there occurred the phrase "the long-assumed negative relationship between protein yield and grain yield". The assumed negative relationship was in fact between protein content and grain yield. 